\title{
Global analysis of DNA methylation in hepatocellular carcinoma by a liquid hybridization capture-based bisulfite sequencing approach
}

Fei Gao ${ }^{2+}$, Huifang Liang ${ }^{1 \dagger}$, Hanlin Lu², Junwen Wang ${ }^{2}$, Meng Xia ${ }^{1}$, Zhimei Yuann², Yu Yao ${ }^{2}$, Tong Wang ${ }^{2}$, Xiaolong Tan ${ }^{1}$, Arian Laurence ${ }^{4}$, Hua Xu ${ }^{5}$, Jingjing $Y_{u^{6}}$, Wei Xiao ${ }^{6}$, Wei Chen ${ }^{2}$, Ming Zhou ${ }^{2}$, Xiuqing Zhang ${ }^{2 *}$, Qian Chen ${ }^{3^{*}}$ and Xiaoping Chen ${ }^{1 *}$

\begin{abstract}
Background: Epigenetic alterations, such as aberrant DNA methylation of promoter and enhancer regions, which lead to atypical gene expression, have been associated with carcinogenesis. In hepatocellular carcinoma (HCC), genome-wide analysis of methylation has only recently been used. For a better understanding of hepatocarcinogenesis, we applied an even higher resolution analysis of the promoter methylome to identify previously unknown regions and genes differentially methylated in HCC.

Results: Optimized liquid hybridization capture-based bisulfite sequencing (LHC-BS) was developed to quantitatively analyze 1.86 million CPG sites in individual samples from eight pairs of HCC and adjacent tissues. By linking the differentially methylated regions (DMRs) in promoters to the differentially expressed genes (DEGs), we identified 12 DMR-associated genes. We further utilized Illumina MiSeq combining the bisulfite sequencing PCR approach to validate the 12 candidate genes. Analysis of an additional 78 HCC pairs on the Illumina MiSeq platform confirmed that 7 genes showed either promoter hyper-methylation (SMAD6, IFITM1, LRRC4, CHST4, and TBX15) or hypo-methylation (CCL2O and NQO1) in HCC.

Conclusions: Novel methylome profiling provides a cost-efficient approach to identifying candidate genes in human HCC that may contribute to hepatocarcinogenesis. Our work provides further information critical for understanding the epigenetic processes underlying tumorigenesis and development of HCC.
\end{abstract}

Keywords: DNA methylation, Liquid hybridization capture-based bisulfite sequencing, Hepatocellular carcinoma

\section{Background}

Hepatocellular carcinoma (HCC) represents an endemic burden worldwide. Well-known risk factors associated with HCC include chronic hepatitis B virus (HBV) and hepatitis $\mathrm{C}$ virus (HCV) infections and toxic, metabolic, and immune-related conditions [1].

\footnotetext{
*Correspondence: zhangxq@genomics.cn; C_Q_N@yahoo.com; chenxp@ medmail.com.cn

${ }^{\dagger}$ Equal contributors

${ }^{2}$ Science \& Technology Department, BGI-Shenzhen, Shenzhen 518083 Guangdong, China

${ }^{3}$ Division of Gastroenterology, Department of Internal Medicine, Tongji Hospital, Tongji Medical College, Huazhong University of Science and Technology, Wuhan 430030 Hubei, China

'Hepatic Surgery Centre, Tongji Hospital, Tongji Medical College, Huazhong University of Science and Technology, Wuhan 430030 Hubei, China Full list of author information is available at the end of the article
}

The development of HCC is a multistep process characterized by the accumulation of genetic mutations and epigenetic aberrations. Epigenetic alterations such as aberrant methylation and histone modification occur far more frequently than genetic mutations in cancers and can significantly affect the efficacy of messenger RNA (mRNA) synthesis without changing the primary DNA sequence [2]. Identification of specific DNA methylation signatures thus has great potential to generate diagnostic markers for early disease detection and further the development of therapeutic regimens.

In mammalian cells, DNA methylation occurs at the $5^{\prime}$ position of the cytosine ring within CpG dinucleotides, via the addition of a methyl group, to create a 5methylcytosine $(\mathrm{m} 5 \mathrm{C})$. DNA methylation sites tend to 
cluster in regions of large repetitive sequences, called CpG Islands (CGIs) [3]. The two most common forms of aberrant CpG methylation in cancer have been widely studied, namely global hypo-methylation that causes chromosomal instability and promoter hypo- or hypermethylation that leads to inappropriate activation of oncogenes or silencing of tumor suppressor genes (TSGs), respectively $[3,4]$.

A number of powerful technologies have emerged in recent years that allow high-throughput detection of genome-wide epigenetic changes in $\mathrm{HCC}$, furthering our understanding of the impact of altered DNA methylation on hepatocarcinogenesis [5-11]. For instance, promoter microarray-based approaches include the methylated CpG island amplification microarray chip (MCAM-chip) that utilizes enzymatic digestion $[5,6]$ and the methylated DNA immunoprecipitation microarray chip (MeDIP-chip) that employs antibody pulldown [12] to enrich methylated DNA. This is followed by profiling on a promoter array and generally results in approximately 25,000 human promoters being analyzed per sample. The second approach relies on bead arrays, which are characterized by bisulfite conversion of DNA followed by the use of a microbeadbased microarray. The highest throughput achieved by this technique to date was the mapping of more than 485,000 CpG sites in HCC through an Infinium 450K array $[10,11]$. Studies that have utilized currently available genome-wide profiling techniques have reported numerous differentially methylated genes in HCC, including tumor suppressor genes. Although many of the genes identified in these investigations have differed, some consistencies have been reported. For example, two studies identified KLHL35, PAX5, PENK, and SPDYA to be hypermethylated in $\mathrm{HCC}$ of viral etiology $[6,9]$, while independent studies have also found IGFALS $[8,13]$ and $M T 1 G[8,13]$ to be repressed by hyper-methylation in HCC. However, there still remains no general consensus as to which genes consistently show differential methylation in HCC. In part, this may be due to intra- or intertumor heterogeneity, differences between studies in the etiology underlying the $\mathrm{HCC}$, or differences in the technique and detection sites used, highlighting the necessity for additional investigations to identify those genes that most consistently show aberrant methylation. An important limitation of previous studies is that none have been able to detect all CpG sites in the entire promoter regions and thereby map the promoter methylome of human HCC. In order to address this shortcoming, we have enhanced the coverage to include promoter regions genomewide and attempted to identify promising methylation markers or characteristic driver genes that may not have been reported previously in HCC.

We previously developed a liquid hybridization capturebased bisulfite sequencing (LHC-BS) technique suitable for
CpG methylation analysis using a massive parallel se quencer-based approach, which relies on specific capture of target regions by liquid hybridization. We demonstrated that this approach could be used to examine the human exome [14] as well as the promoter methylome [15]. In the present study, we initially performed promoter-targeted LHC-BS on eight paired HCC tissues to analyze 1.86 million CpG sites located at the promoter regions of 31,372 (91.8 \%) genes. Next, high-depth RNA-sequencing was applied to search for candidate genes in HCCs that showed a negative correlation between gene expression and promoter methylation. Illumina MiSeq combining the bisulfite sequencing PCR approach was further carried out to validate these candidate genes in an additional 78 HCC tumor and non-tumor pairs. Using this approach, we confirmed that 7 genes showed altered promoter methylation in HCC, with SMAD6, IFITM1, LRRC4, CHST4, and TBX15 exhibiting promoter hyper-methylation, and CCL2O and NQO1 exhibiting promoter hypo-methylation. Western blot and quantitative real time polymerase chain reaction (qRT-PCR) experiments confirmed that a total of 5 genes showed altered expression in HCC samples. Therefore, LHC-BS-based promoter methylome analysis in HCC represents an effective technique for assessing epigenetic changes across the human genome.

\section{Results}

The promoter methylome differentiates tumor tissue from adjacent non-tumor tissue in HCC

The clinicopathologic features of the 8 patients with HCC in this promoter-wide methylation study are described in Additional file 1: Table S1. The primary etiology of this group was HBV infection (7 of 8 patients). All patients had a single tumor and most of the primary tumors (5 of 8 ) had moderately differentiated histology; 6 of 8 had stage II tumor, classified using the American Joint Committee on Cancer (AJCC) TNM system.

A LHC-BS approach $[14,15]$ was subsequently applied to profile the promoter methylome of the 8 sample pairs. Promoters were denoted as regions from $-2200 \mathrm{bp}$ to $+500 \mathrm{bp}$ of the transcriptional start sites (TSS) [16]. Based on the hg19 reference human genome, a total of 150,407 capture probes from the Crick strand were customized, capturing 1.86 million CpG nucleotides in the promoters. Based on this design, the Watson strand can be captured, enabling a coverage of 31,372 (91.8 \%) genes in the RefSeq database [15]. We obtained an average of $4.4 \mathrm{~Gb}$ clean data for each sample, reaching $23 \times$ read depth, of which $94.77 \%$ were mapped to at least one genomic position, with $87.75 \%$ mapped uniquely to the reference genome. Furthermore, $94.21 \%$ of the uniquely mapped reads were located at the defined promoter regions (Additional file 2: Table S2). We then filtered out all the $\mathrm{CpG}$ sites with less than $4 \times$ coverage in the 8 paired samples. The median value of 
CpG coverage between the lowest $(994,997)$ and highest $(1,685,393)$ sample was 1.374799 million CpGs.

To identify differential methylation of CpG loci linked to HCC, we further picked 690,858 CpG sites achieving a minimum read coverage of $4 \times$ in all 16 samples and performed a hierarchical clustering analysis. Based on the average level of methylation crossing downstream 500-bp region around TSS, the tumor could be clearly separated from adjacent non-tumor by significant changes in the pattern of the promoter methylome (Fig. 1a). Principal component analysis (PCA) consistently demonstrated that HCC tissue exhibited greater variance than non-tumor tissue, and this was further confirmed by chi-square tests (Additional file 3: Figure S1A, B). These findings may suggest generalized disruption of the integrity of the methylome in $\mathrm{HCC}$.

The promoter regions of genes losing or acquiring DNA methylation show different $\mathrm{CpG}$ contents in $\mathrm{HCC}$

To analyze the relationship between promoter DNA methylation and activity determined by the CpG content of the promoters, we applied a previously described classification of promoters as having either high-CpG content (HCP), intermediate CpG content (ICP), or low-CpG content (LCP), based on the $\mathrm{CpG}$ ratio, GC content, and length of the CpG-rich region [16] (Additional file 4: Figure S2A). In line with a previous report using MeDIP technology, our analysis demonstrated that genes acquiring low DNA methylation levels in tumors were mostly characterized by the presence of $\mathrm{HCP}$ promoters (Additional file 4: Figure
S2B). We further performed hierarchical clustering analyses of CGIs, and a chi-square analysis was then applied to select the top 1000 genes containing highly variable CGI methylations based on the $P$ values (Fig. 1b). In general, many of these 1000 genes had a substantially higher methylation ratio in tumor tissue than in non-tumor tissue (Fig. 1b). Interestingly, the majority of these 1000 CGIs were consistently hypo-methylated in poorly differentiated tumor (ID NO. 388) compared with moderately or welldifferentiated tumors. Although one tumor (ID NO. 734) clustered closely with its adjacent tissue, we suspect that this may have been due to contamination of the tumor sample with non-tumor tissue. Overall, the data support the possibility that enriched HCPs may be responsible for inhibiting the expression of the corresponding genes.

\section{Comparisons of promoter $\mathrm{CpG}$ methylation between HCC tissue and adjacent tissue reveal differentially methylated regions and DEGs}

We next applied a pair-wise comparison to reveal differentially methylated regions (DMRs). In each comparison, the sliding window strategy was used to determine if the region within the window exhibited differential methylation between tumor and non-tumor samples (Additional file 5: Material and Methods). The approach generated an average of 2972 DMRs for 16 samples, although there was variation between sample pairs, suggesting high intratumor heterogeneity in DNA methylation (Additional file 6: Figure S3B). However, 77 genes with one or two DMRs were found in 6 of the 8 paired samples, and $67.5 \%$ of
A

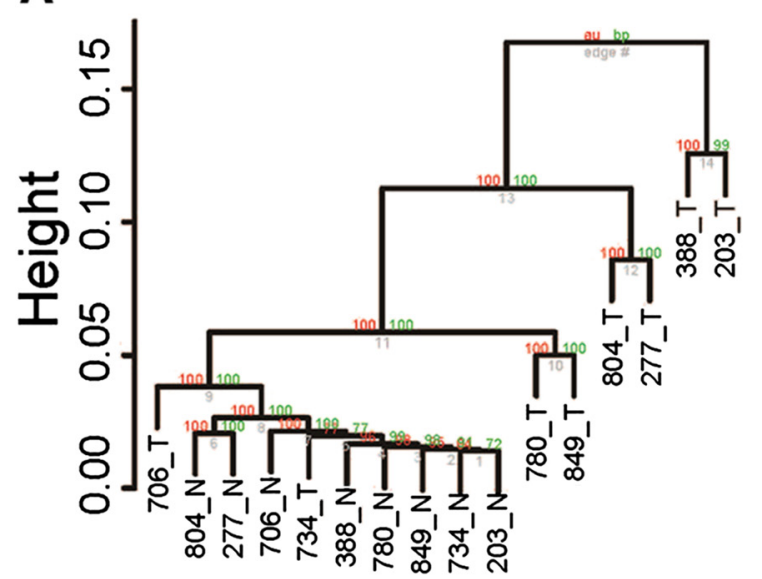

B methylation ratio

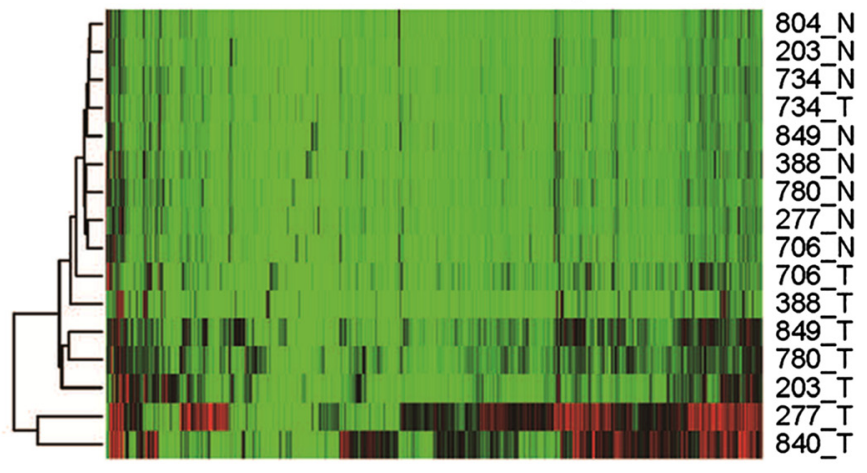

Fig. 1 Hierarchical clustering analyses of the promoter methylomes of 8 pairs of HCC and adjacent non-tumor samples. a Clustering of the average DNA methylation levels of all promoters were used in the "Pvclust" algorithm. Two types of $P$ values (\%) on the edge of the cluster are provided: approximately unbiased (AU) $P$ value and bootstrap probability (BP) $P$ value, which indicate how well the cluster is supported by the data. $\mathbf{b}$ Clustering of the top 1000 CGls containing highly variable methylations that were selected based on $P$ values from a chi-square analysis. The methylation ratio was calculated as sequenced reads number of C/sequenced reads number of C $+\mathrm{T}$. Red color indicates high methylation ratio, black color indicates moderate methylation ratio, and green color indicates low methylation ratio._T denotes tumor tissue samples, and _N denotes non-tumor tissue samples 
these DMRs were hyper-methylated in the tumor tissue (Additional file 7: Table S3).

Promoter CGI methylation has frequently been associated with silencing of gene expression. To obtain expression data from the $8 \mathrm{HCC}$ pairs, we used Illumina highthroughput RNA-seq technology to assess differentially expressed genes (DEGs). After removing low quality reads, we obtained $84.55 \%$ of reads aligned to previously annotated genes, reaching $78.13 \%$ of mapped unique reads. Our analysis determined 18,850 genes exhibiting at least one unique read. To identify DEGs, we next performed a pair-wise comparison between tumor and nontumor tissue using a fold change cutoff of reads per kb per million (RPKM) values larger than 2 and an FDRadjusted $P$ value less than 0.01 [17]. Using this approach, the median numbers of genes identified as DEGs for the 8 paired samples were 7019 , and the majority showed down-regulated expression in HCCs (Additional file 6: Figure S3A). Only 93 DEGs were shared by 6 of the 8 paired samples (Additional file 8: Table S4).

We hypothesized that there would be a relationship between the presence of DMRs in specific promoters and the DEGs in the liver tumors. As a result, 24 genes containing DMRs in promoter regions were subsequently matched and met the selection criteria in at least 5 of the 8 sample pairs (Additional file 9: Table S5). Among these, 20 genes showed expression levels negatively associated with the DMR methylation status. These included 4 genes hypo-methylated in tumor tissue (CLCNKA, BAIAP2L2, CCL20, and NQO1) and 16 genes hyper-methylated in tumor tissue (IFITM1, SMAD6, TBX15, CHST4, LRRC4, PHYHD1, STEAP4, TACSTD2, NPC1L1, THRSP, KCNJ10, PALM3, FAM134B, TMEM100, $P M 20 D 1$, and GRHL2).

\section{Selection of candidate genes and validation of methylation in 78 pairs of HCCs by MiSeq-BSP}

We further acquired an additional 78 paired samples (of $\mathrm{HCC}$ and adjacent tissue) to validate the genes initially identified in the LHC-BS study. Since most of the primary tumors studied in the LHC-BS analysis had a well or moderately differentiated histology, we obtained 39 well-to-moderately and 39 moderately differentiated HCCs together with their matched adjacent tissues (Additional file 10: Table S6). The majority of the patients $(96 \%)$ in our study were male; the average age at diagnosis and treatment of HCC was $47.6 \pm 10.1$ years; $88 \%$ had HBV infection. With regard to the common factors associated with HCC prognosis and recurrence, $83 \%$ of the subjects had a single tumor, $65 \%$ of the primary tumors were more than $5 \mathrm{~cm}$ in diameter, $58 \%$ of patients had stage III tumors, and $41 \%$ of patients had blood alpha-fetoprotein (AFP) levels greater than $4000 \mathrm{ng} / \mathrm{ml}$. Therefore, the subjects represent a group of patients with hyper-vascular primary liver malignancy, who have a poor prognosis, associated with large tumor size as well as involvement of nearby or major vessels.

By undertaking a comprehensive literature search on liver carcinogenesis, we manually selected 12 of these 20 genes showing an inverse relationship between promoter methylation and gene expression. Among these 12 genes, 10 genes up-regulated in tumors with a hypermethylated promoter (IFITM1, SMAD6, TBX15, CHST4, LRRC4, PHYHD1, STEAP4, TACSTD2, NPC1L1, and THRSP) and 2 genes down-regulated in tumors with a hypo-methylated promoter (CCL2O and NQO1) were further validated using Illumina MiSeq sequencingbased bisulfite sequencing PCR (MiSeq-BSP). Libraries for the 12 genes were prepared and individually barcoded for high-throughput pair-end sequencing using MiSeq2500 (Additional file 5: Material and Methods; Additional file 9: Table S5). Deep-sequencing of individual PCR fragments was achieved in a cost-effective way (Additional file 11: Table S7). We found that 7 genes (IFITM1, SMAD6, TBX15, CHST4, LRRC4, CCL20, and NQO1) showed significantly different promoter methylation levels between tumor and non-tumor tissue $(P$ value $<0.001)$ in approximately $80 \%$ of the 78 HCCs (Fig. 2a). In addition, 20$40 \%$ of the examined HCCs showed a minimal difference in the mean values of 0.2 (corresponding to a $20 \%$ difference in methylation) (Fig. 2b), indicating a highly tumorspecific promoter methylome change in these genes. We further performed supervised PCA on these 7 genes, which clearly separated tumors from nontumors (Fig. 2c). However, the methylation status of these genes was not associated with any of the clinicopathologic findings, including histological differentiation and TNM stage (Fig. 2d).

\section{Validation of candidate gene expression in HCCs}

Among the genes that exhibited aberrant methylation in HCCs, SMAD6 has at least two transcript variants, the full-length variant 1 (NM_005585.4) and the short variant 2 (NM_001142861.2). Genomic sequence alignment suggested that the promoter hyper-methylation observed occurred in the shorter spliced form, which lacks one inframe exon compared with the full-length transcript, variant 1 (Fig. 3a). We further chose the primer pair specific for variant 2 and examined its expression in $8 \mathrm{HCC}$ pairs assessed by the LHC-BS assay. Through qRT-PCR analysis, we confirmed reduced SMAD6 variant 2 mRNA expressions in all examined tumors, indicating HCCspecific down-regulation of variant 2 (Fig. 3b).

Western blot analysis was further performed on the 8 HCC pairs to confirm the protein expression of the candidate genes, including IFITM1, CHST4, TBX15, LRRC4, and NQO1. Compared with adjacent non-tumor tissue, we observed reduced protein expression of IFITM1 in 6 


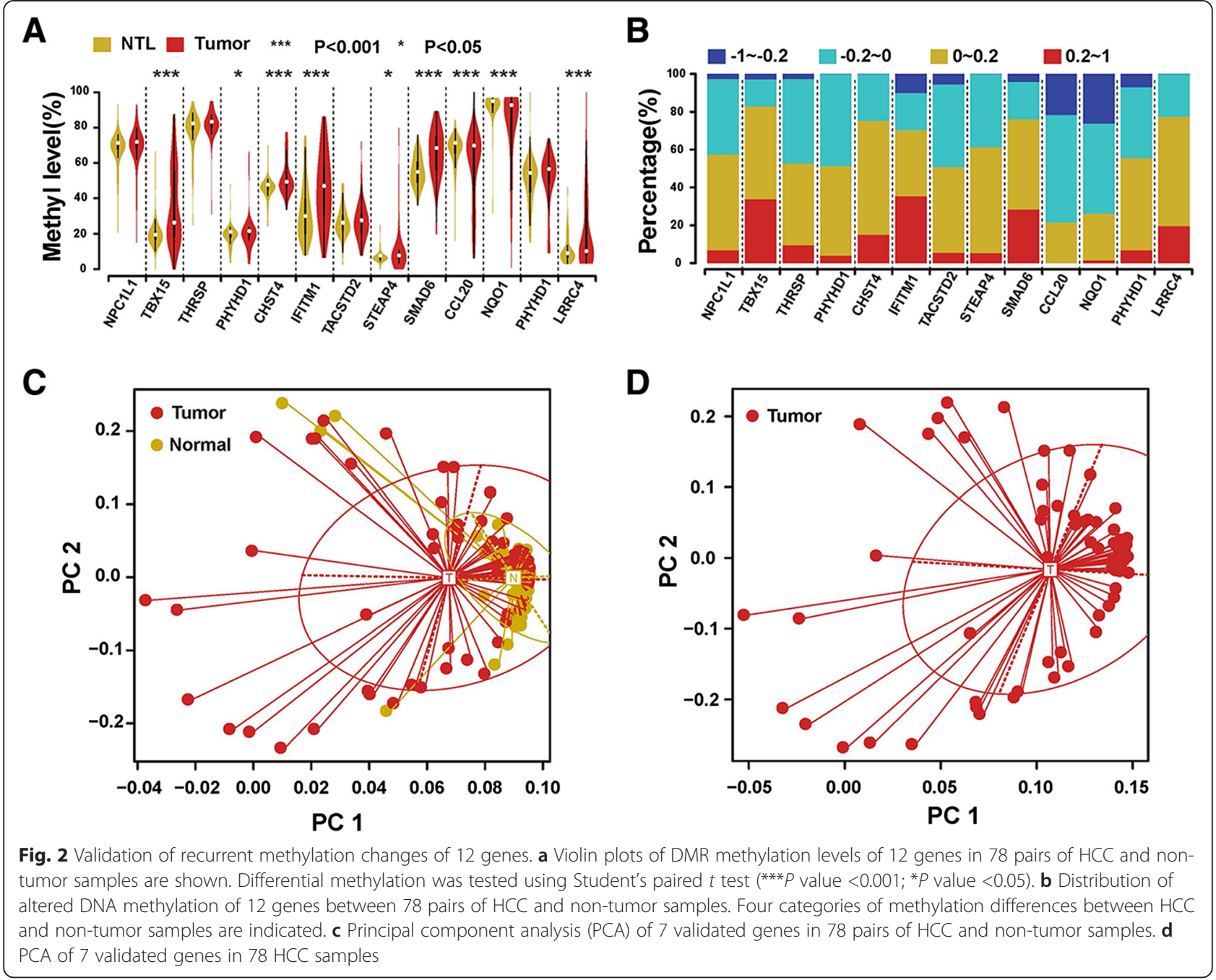

of 8 tumors, lower TBX15 levels in 7 of 8 tumors, and decreased CHST4 amounts in 5 of 8 tumors (Fig. 3c, d). However, we could not detect alterations in the protein expressions of LRRC4 and NQO1 in HCC tissue (data not shown).

\section{Demonstration of epigenetic regulation of candidate} genes transcription via demethylation assays in cell lines DNMT1 and DNMT3B, which belong to the DNA methyltransferase (DNMT) family, control DNA methylation. To further evaluate the impact of promoter methylation on gene expression, we utilized two cancer cell lines, namely HCT116 wild type and HCT116 ${ }^{\text {DNMT1-/- DNMT3B-/- }}$ double knockout (DKO) cells [18]. A total of 5 of 6 genes, including CHST4, IFITM1, TBX15, LRRC4, and SMAD6 variant 2, showed high promoter methylation levels (>80\%) in HCT116, while more than $50 \%$ of their methylation was lost in DKO cells as a consequence of DNMT inhibition. Correspondingly, CCL20, CHST4, IFITM1, and SMAD6 variant 2 showed elevated expression in HCT116
DKO, confirmed by qRT-PCR (Fig. 4a and Additional file 12: Table S8).

For hyper-methylated or transcriptionally silenced genes, the DNA demethylating agent 5-aza-2-deoxycytidine (DAC) is known to restore gene expression $[19,20]$. We further analyzed candidate gene expression upon DAC treatment in two immortalized non-tumor liver cell lines (QSG-7701 and HL-7702) and two HCC cell lines (HLE and HLF). For CCL20, we observed elevated expression in HCC cell lines upon treatment with DAC, but not in non-tumor cell lines, suggesting that CCL2O methylation status may be specifically associated with HCC prognosis. In agreement with the observations made in the HCT116-DKO study, IFITM1 expression in HCC cell lines was restored after treatment with DAC (Fig. 4b). However, TBX15, LRRC4, and CHST4 showed no systematic difference in expression between HCC and non-tumor liver cell lines (Additional file 13: Figure S4). Nonetheless, these observations in cell lines do not exclude the possibility that the expressions of TBX15 and 

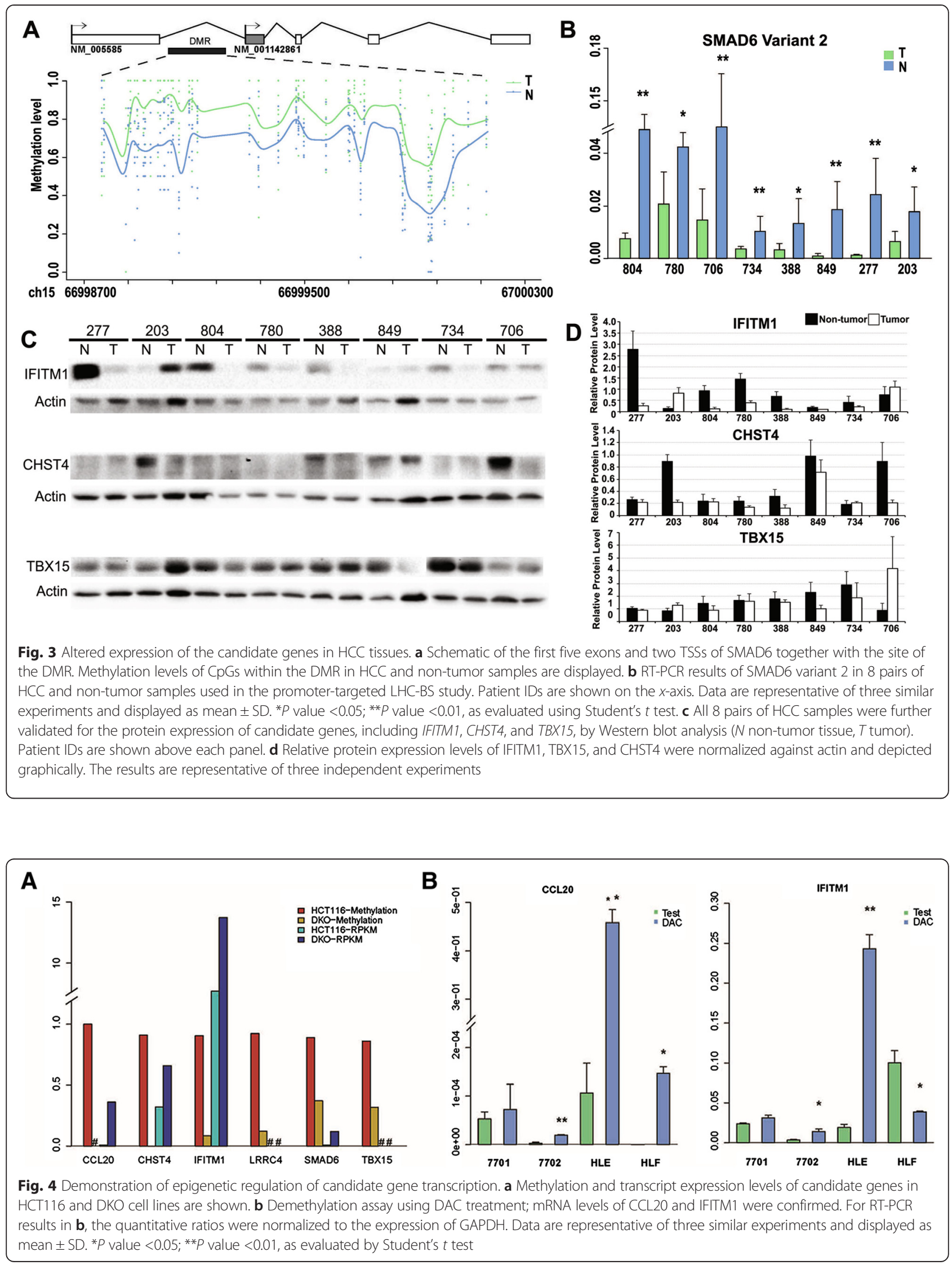
CHST4 are altered in some patients with HCC due to promoter hyper-methylation, particularly as the Western blot analysis of the 8 paired samples described above revealed that TBX15 levels were reduced in 7 of 8 tumors, and CHST4 levels were decreased in 5 of 8 tumors (Fig. 3c, d).

Taken together, our results suggest that SMAD6 variant 2, IFITM1, TBX15, and CHST4 may act as TSGs in HCC that are silenced by promoter hyper-methylation; meanwhile, CCL2O may be epigenetically activated in tumor through promoter hypo-methylation, and elevated expression may be associated with a poor prognosis in HCC.

\section{Discussion}

HCC is a genetically heterogeneous disease. The goal of genomic and epigenetic profiling efforts in studies of $\mathrm{HCC}$ is to identify characteristic driver genes and improve our understanding of the etiology of the disease. Promoter $\mathrm{CpG}$ islands with aberrant hyper-methylation are recognized as being an important mechanism for inactivation of tumor-related suppressor genes in human cancers. Although this has been extensively studied in colon cancer, with many genes identified as harboring altered methylation in their promoter CGIs [21], there is currently far less information regarding HCC. Our previous study showed that the novel LHC-BS approach is a reliable and efficient analytical platform for generating a single-base-pair resolution methylome map of promoter regions in cancer and normal cell lines [14, 15]. In the current study, we further optimized the technology to profile the promoter methylome in 8 paired HCC samples.

The flow chart in Fig. 5 describes the strategy we used to identify HCC-specific candidate genes. We first identified 77 genes with one or two DMRs shared by 6 of the 8 liver tumors and then detected 93 DEGs that were common to the tumors compared with non-tumor liver tissue (Additional file 7: Table S3; Additional file 8: Table S4). We further integrated these data and matched DEGs to DMRs. The approach allowed us to characterize 20 genes showing an inverse relationship between $\mathrm{CpG}$ methylation and transcriptional activity. Although we found greater heterogeneity in the DMR profiles among tumors as compared with non-tumors, our findings further support the idea that tumors in general have highly heterogeneous DNA methylation patterns. As a result of the limited sample size and strict criteria, we excluded low quality variants. Therefore, it should not be surprising that the current study has not yielded many overlapping candidate genes with altered promoter methylation. Indeed, to focus on genes for which differential methylation and expression could be related, we applied larger sample cohorts (78 paired HCC samples) for a further validation using Illumina MiSeq-BSP (Fig. 2). Using this approach, 7 genes were identified as undergoing tumor-

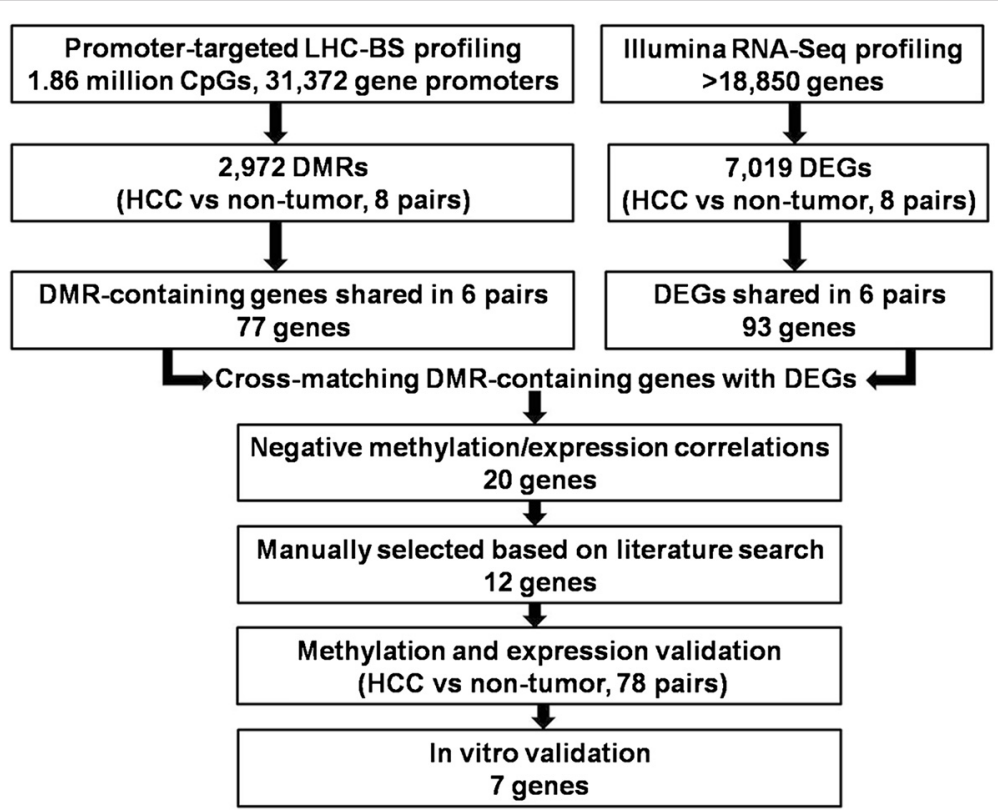

Fig. 5 Flow chart. Eight pairs of HCC samples were used to screen for candidate methylation markers using the promoter-targeted LHC-BS approach. In HCC tissues, 2972 DMRs were determined and 77 genes with one or two DMRs were found to be common in 6 of 8 paired samples. Gene expression was analyzed using the Illumina high-throughput RNA-seq technology, and 7019 DEGs were detected. Among them, 93 DEGs were shared by 6 of the 8 paired samples. Through cross-matching DMR-containing genes with DEGs and searching the literature, 20 genes were selected for validation. Twelve candidate genes were validated for methylation and gene expression in 78 paired HCC samples. Functional validation was performed in vitro, and 7 genes were identified as candidate genes in HCC, whose altered expression may contribute to hepatocarcinogenesis 
specific aberrant promoter methylation in $\mathrm{HCC}$, including IFITM1, SMAD6, TBX15, CHST4, and LRRC4 with hyper-methylated promoters and CCL2O and NQO1 with hypo-methylated promoters.

We subsequently confirmed the correlation between promoter methylation and expression levels of the SMAD6, IFITM1, TBX15, CHST4, and CCL20 genes (Figs. 3 and 4). In particular, we found that IFITM1 showed significantly down-regulated expression with abnormal epigenetic regulation in HCC. IFITM1 encodes an interferon (IFN)-induced antiviral protein that plays a key role in IFN-gamma mediated anti-proliferation either by inhibiting ERK activation or inducing p53dependent G1 arrest [22]. The down-regulation of IFITM1 has been linked to both low-grade diffuse astrocytomas and breast cancer [23, 24]. In addition, epigenetic silencing of the IFITM1 protein has been found in other human malignancies, including gastric cancer [25]. Given that IFN-gamma plays a role during HCC immunotherapy and has a direct inhibitory effect on $\mathrm{HCC}$ by inducing apoptosis, future studies will be focused on understanding whether IFITM1 suppresses HCC through IFN-gamma signal transduction.

Another TSG candidate highlighted is the short transcript of SMAD6, variant 2, but not the full-length $S M A D 6$, variant 1. SMAD transcription factors lie at the core of the transforming growth factor-beta (TGF- $\beta$ ) signaling pathway. Recent studies have suggested that differentially spliced forms of many SMAD family members are generated by genetic or epigenetic inactivation and may represent an important step in neoplastic transformation [26-28]. As noted, tumor-derived variants of SMADs often carry a mutated $\mathrm{N}$ - or Cdomain, which inhibits the formation of homodimers or heterodimers with other SMADs, resulting in a loss of sensitivity to TGF- $\beta$ cytokine family-mediated growth arrest [26, 27]. Unlike SMAD6 variant 1 , which is broadly expressed and functions as a negative regulator of bone morphogenetic protein (BMP) and TGF- $\beta$ signaling [29], variant 2 has a truncated MAD homology (MH)-1 domain and is variably expressed. It has been reported that variant 2 forms non-productive heterodimers with SMAD7 in a transcriptional complex, thereby, interfering with conventional BMP/TGF- $\beta 1$ signaling [30]. Additional studies are required to determine whether silencing SMAD6 variant 2 may contribute to $\mathrm{HCC}$ tumorigenesis.

Treatment of HCC cell lines with DAC was associated with CCL20 promoter hypo-methylation together with elevated expression (Fig. 4). Up-regulated CCL2O expression is observed in many tumors including HCC [31]. The oncogenic role of CCL2O has been characterized to be the promotion of tumor cell invasion through the upregulation of MMP-9 in colorectal cancer cells and pancreatic adenocarcinoma [32, 33]. Our findings suggest that the presence of CCL20 in the tumor may indicate a poor prognosis of HCC. Further studies are required to completely understand the epigenetic and molecular mechanisms regulating CCL20 expression and determine whether it plays a role in metastasis in advanced $\mathrm{HCC}$.

However, HBV infection is the major risk factor for HCC in China. In this study, 7 of 8 patients were infected with HBV, including tumor and adjacent liver tissues (primarily cirrhotic). The current study could not confirm an association of these genes with HBV infection and HBV-induced HCC tumorigenesis. Further studies are warranted to clarify these issues.

\section{Conclusions}

In summary, to the best of our knowledge, this is the first report to apply promoter-targeted LHC-BS technology to assessing the promoter methylome in $\mathrm{HCC}$ at a single-base resolution. Our current analysis focused on differential methylation patterns in or near gene promoters. In combination with RNA-seq and gene expression data, this technique allows for the identification of promising tumor suppressor and oncogene candidates in human HCC. Our work highlights the potential of costefficient epigenetic approaches in the prevention and therapy of human HCC.

\section{Methods}

\section{Patients and specimens}

This study was approved by the Institutional Review Board of Tongji Hospital, Tongji Medical College of Huazhong University of Science and Technology (HUST) and the local ethics committee in Hubei province, China. All patients included in the study were referred for treatment at Tongji Hospital between 2008 and 2012. Written informed consent was obtained from each patient. The histological diagnosis and classification of HCC and adjacent liver tissue (primarily cirrhotic) were performed by experienced pathologists. Information about risk factors and other clinicopathologic characteristics for HCC was retrieved from medical records. Among these, HBV (hepatitis B surface antigen; HBsAg) and HCV (anti-HCV) statuses were determined by immunoassays.

\section{Promoter-targeted LHC-BS and RNA-seq}

Promoter-targeted LHC-BS was performed as described previously [15]. Briefly, $1 \mu \mathrm{g}$ DNA per sample was processed by fragmentation, blunt end repair, 3'adenylation, and 5'-methylcytosine index adapter ligation. Then, $250 \mathrm{ng}$ DNA from each offer adapter-ligated libraries were pooled together for the liquid hybridization capture procedure. We applied capture program, bisulfite treatment, and PCR amplification based on previous protocols [15]. 
For RNA-seq [34], the poly(A)-containing mRNA was purified using Oligo(dT) Beads (Illumina), and this was followed by fragmentation. The converted doublestranded cDNA product was subjected to blunt-ending, $\mathrm{dA}$ addition to the $3^{\prime}$ end and adapter ligation. The adapter-ligated fragments were size selected $(200 \pm 20 \mathrm{bp})$ using $2 \%$ TAE-Certified Low-Range Ultra Agarose (BioRad). After purification, 15 rounds of PCR amplification were performed to enrich the adapter-ligated cDNA libraries. The LHC-BS and RNA-seq libraries were performed on an Agilent Technologies 2100 Bioanalyzer using the Agilent DNA 1000 chip kit. They were subsequently quantified on a StepOne plus qPCR, and library products were sequenced using the Illumina Hiseq2000.

\section{Computational processing of the next-generation sequencing data}

See Additional file 5: Materials and Methods.

\section{5'-aza-2'-Deoxycytidine treatment of cell lines}

Two human HCC cell lines (HLE and HLF) and 2 immortalized liver cell lines (QSG-7701 and HL-7702) were cultured at $5 \% \mathrm{CO}_{2}, 37{ }^{\circ} \mathrm{C}$, and $95 \%$ humidity in Dulbecco's modified Eagle medium (DMEM; Gibco-Life Technologies) supplemented with $10 \%$ fetal bovine serum (FBS; Gibco; Life Technologies), 100 units $/ \mathrm{mL}$ penicillin, and $100 \mu \mathrm{g} / \mathrm{mL}$ streptomycin (Sigma-Aldrich). After growing to about $60 \%$ confluency, cells were treated with $5 \mu \mathrm{M}$ DAC (Sigma) for $72 \mathrm{~h}$. DAC was replenished every $24 \mathrm{~h}$. After $72 \mathrm{~h}$, DAC-treated cells and untreated controls were harvested.

\section{Illumina MiSeq sequencing-based bisulfite sequencing PCR}

See Additional file 5: Materials and Methods, and Additional file 11: Table S7.

\section{Quantitative real-time PCR}

Reverse transcribed with ReverTra Ace- $\alpha{ }^{-{ }^{m x}}$ (Toyobo) was $1 \mu \mathrm{g}$ of total RNA. qRT-PCR was carried out using TaqMan Universal Master Mix II with UNG on an ABI StepOne Real-Time PCR System (Applied Biosystems, USA). The relative RNA expression was calculated using the delta delta threshold cycle $(\triangle \Delta C T)$ method and normalized to glyceraldehyde-3-phosphate dehydrogenase (GAPDH) expression. Each assay was performed in triplicate.

\section{Western blot}

Western blot was performed with antibodies specific to IFITM1 (mouse monoclonal antibody 1:2000, Proteintech Group, USA), TBX15 (rabbit polyclonal antibody 1:1000, Aviva Systems Biology, USA), CHST4 (rabbit polyclonal antibody 1:1000, Aviva Systems Biology), LRRC4 (rabbit polyclonal antibody 1:1000, Abgent, USA), and NQO1 (mouse monoclonal antibody 1:1000, Santa Cruz Biotechnology, USA). $\beta$-actin (mouse monoclonal antibody 1:10,000, Santa Cruz Biotechnology) was used as a loading control. The expression levels of the proteins were quantified by ChemiDoc ${ }^{\text {TM }}$ MP Imager Universal hood III (BioRad Laboratories Inc., USA).

\section{Statistical analysis}

All differential methylation analyses were performed using $M$ values, and $\beta$ values, ranging from 0 to $100 \%$ methylation. Differential methylation was tested statistically using Student's paired $t$ test. CpG sites with FDR $<0.05$ and a within-pair methylation difference of $\geq 5 \%$ were considered differentially methylated. Moderated $t$ statistics with the Benjamini and Hochberg (BH) correction methods were used to compare within-pair differences of tumor and nontumor pairs between groups, to examine whether the identified within-pair methylation discordances were group specific. Hierarchical clustering analyses were performed on the promoter methylomes of paired samples. Clustering of average DNA methylation levels of all promoters were used in the "Pvclust" algorithm. Two types of $P$ values (\%) on the edge of the cluster are provided: approximately unbiased (AU) and bootstrap probability (BP) $P$ values. The top 1000 CGIs containing highly variable methylations were selected based on $P$ values from a chi-square analysis. $\mathrm{R}$ and Stata statistical software (release 12.0; Stata Corporation, USA) were used for statistical analysis.

\section{Gene set and pathway analyses}

The significance of predefined sets of CpGs, each set representing a pathway on KEGG, was analyzed by the R package GSA. GSA was applied on within-pair differences in methylation and run with 1000 permutations. An FDR cutoff of 0.1 and a $P$ value cutoff of 0.05 were considered significant IPA (Ingenuity Systems, Redwood City, CA, USA), with KEGG pathways used to generate gene networks and functions in $\mathrm{HCC}$ tumorigenesis and development.

\section{Data and material availability}

All raw and processed data of promoter LHC-BS and RNA-Seq have been deposited in NCBI's Gene Expression Omnibus (GEO) with accession reference GSE55759.

\section{Additional files}

Additional file 1: Table S1. Clinicopathology features of patients with HCC studied for genome-wide promoter methylation analysis.

Additional file 2: Table S2. Data generation for LHC-BS and RNA-Seq.

Additional file 3: Figure S1. Tumors are separated from adjacent nontumors through analysis of the patterns of the promoter methylomes. A, principal component analysis (PCA) of the average methylation levels of total promoters in 8 pairs of HCCs and non-tumor samples; B, cumulative curves of $-\log (P$ value $)$ from the chi-square test on intra-group variations of CpG methylation levels in HCC tumors (T) and non-tumors (N). 
Additional file 4: Figure S2. Promoter classification based on CpG representation. A, the histograms represent the distribution of observed versus expected $\mathrm{CpG}$ frequencies for all promoters, displaying the low (LCPS, red), intermediate (ICPs, green), and high (HCPs, blue) CpG content promoters; $B$, the proportion of the three categories of promoters.

Additional file 5: Materials and Methods. Computational processing of the next-generation sequencing data; Illumina MiSeq sequencingbased bisulfite sequencing PCR (MiSeq-BSP).

Additional file 6: Figure S3. Percentage of DEGs and DMR-containing genes determined in 8 paired HCC tissues. A, counts of down- and upregulated genes in HCCs in comparison with non-tumors; $B$, counts of hyper- and hypo-methylated genes in HCCs in comparison with nontumor tissue.

Additional file 7: Table S3. Summary of 77 genes containing DMRs across at least 6 pairs of HCCS and non-tumors by LHC-BS.

Additional file 8: Table S4. Summary of 93 DEGs shared by at least 6 paired HCC tissues.

Additional file 9: Table S5. Summary of 20 genes showing negative correlation between methylation and gene expression in at least 5 out of 8 pairs of samples.

Additional file 10: Table S6. Clinicopathological features of 78 Chinese HCC patients studied for validation of gene expression.

Additional file 11: Table S7. Primer sequences for MiSeq-BSP and RTPCR validation.

Additional file 12: TableS8. Coverage and methylation levels of 12 genes validated by MiSeq-BSP

Additional file 13: FigureS4. RT-PCR results for TBX15, LRRC4 and CHST4 in a demethylation assay. The quantitative ratios were normalized to the expression of GAPDH. Data are representative of three similar experiments and displayed as mean \pm SD. ${ }^{*}, P<0.05$ as evaluated by Student's $t$ test.

\section{Abbreviations}

AFP: alpha-fetoprotein; BMP: bone morphogenetic protein; CGI: CpG Islands; DAC: 5-aza-2-deoxycytidine; DEG: differentially expressed genes; DMR: differentially methylated regions; DNMTs: DNA methyltransferases; HBV: Hepatitis B virus; HCC: hepatocellular carcinoma; HCP: high-CpG promoters; HCV: Hepatitis C virus; ICP: intermediate-CpG promoters; LCP: low-CpG promoters; LHC-BS: liquid hybridization capture-based bisulfite sequencing; MH: MAD homology; MiSeq-BSP: MiSeq sequencing-based bisulfite sequencing PCR; PCA: principal component analysis; RPKM: reads per kb per million; TSG: tumor suppressor gene.

\section{Competing interests}

The authors declare that they have no competing interests.

\section{Authors' contributions}

The experiments were conceived and designed by FG and QC. The experiments were performed by HFL, JWW, and MX. The data were analyzed and interpreted by FG, HFL, ZMY, YY, TW, and XLT. HX, JJY, WX, WC, and MZ participated in the conduction and coordination of the study, including providing cell lines and reagents, acquiring and managing patients, and providing facilities. The study was supervised by XQZ, QC and XPC. The manuscript was written by FG, AL, and QC. All authors read and approved the final manuscript.

\section{Acknowledgements}

We are grateful to all the staff of the Hepatic Surgery Center at Tongji Hospital in Wuhan for providing valuable samples and clinical information. The authors thank Arian Laurence at the Newcastle upon Tyne Hospitals NHS Foundation Trust and Massimo Gadina at National Institute of Health for English language editing. The authors would like to acknowledge the following funding sources: the Major and Special Program of National Science and Technology in Twelfth Five-year Plan of China (2012ZX10002016-004, 2012ZX10002010-001-004, XPC), Major Science Foundation of the Ministry of Health of China (201302009, XPC), National Natural Science Foundation of China (31200666, 81471612, QC; 81202300,
HFL and 81372495, XPC), Chinese 863 Program (2012AA02A201, XQZ), and Innovative R\&D Team Program of Guangdong Province (2009010016, XQZ).

\section{Author details}

${ }^{1}$ Hepatic Surgery Centre, Tongji Hospital, Tongji Medical College, Huazhong University of Science and Technology, Wuhan 430030 Hubei, China. ${ }^{2}$ Science \& Technology Department, BGI-Shenzhen, Shenzhen 518083 Guangdong, China. ${ }^{3}$ Division of Gastroenterology, Department of Internal Medicine, Tongji Hospital, Tongji Medical College, Huazhong University of Science and Technology, Wuhan 430030 Hubei, China. ${ }^{4}$ The Newcastle upon Tyne Hospitals NHS Foundation Trust, Freeman Hospital, Freeman Road, High Heaton, Newcastle upon Tyne NE7 7DN, UK. ${ }^{5}$ Department of Urology, Tongji Hospital, Tongji Medical College, Huazhong University of Science and Technology, Wuhan 430030 Hubei, China. ${ }^{6}$ Translational Medicine Center, Tongji Hospital, Tongji Medical College, Huazhong University of Science and Technology, Wuhan 430030 Hubei, China.

Received: 5 May 2015 Accepted: 3 August 2015

Published online: 21 August 2015

\section{References}

1. Gomaa Al, Khan SA, Toledano MB, Waked I, Taylor-Robinson SD. Hepatocellular carcinoma: epidemiology, risk factors and pathogenesis. World J Gastroenterol. 2008;14(27):4300-8.

2. Consortium EP, Bernstein BE, Birney E, Dunham I, Green ED, Gunter C, et al. An integrated encyclopedia of DNA elements in the human genome. Nature. 2012;489(7414):57-74. doi:10.1038/nature11247.

3. Plass C. Cancer epigenomics. Hum Mol Genet. 2002;11(20):2479-88.

4. Tischoff I, Tannapfe A. DNA methylation in hepatocellular carcinoma. World J Gastroenterol. 2008;14(11):1741-8.

5. Gao W, Kondo Y, Shen L, Shimizu Y, Sano T, Yamao K, et al. Variable DNA methylation patterns associated with progression of disease in hepatocellular carcinomas. Carcinogenesis. 2008;29(10):1901-10. doi:10.1093/ carcin/bgn 170.

6. Shitani M, Sasaki S, Akutsu N, Takagi H, Suzuki H, Nojima M, et al. Genomewide analysis of DNA methylation identifies novel cancer-related genes in hepatocellular carcinoma. Tumour Biol. 2012;33(5):1307-17. doi:10.1007/ s13277-012-0378-3.

7. Hernandez-Vargas H, Lambert MP, Le Calvez-Kelm F, Gouysse G, McKayChopin S, Tavtigian SV, et al. Hepatocellular carcinoma displays distinct DNA methylation signatures with potential as clinical predictors. PLoS One. 2010;5(3):e9749. doi:10.1371/journal.pone.0009749.

8. Neumann O, Kesselmeier M, Geffers R, Pellegrino R, Radlwimmer B, Hoffmann K, et al. Methylome analysis and integrative profiling of human HCCs identify novel protumorigenic factors. Hepatology. 2012;56(5):1817-27. doi:10.1002/hep. 25870

9. Shen J, Wang S, Zhang YJ, Kappil M, Wu HC, Kibriya MG, et al. Genomewide DNA methylation profiles in hepatocellular carcinoma. Hepatology. 2012;55(6):1799-808. doi:10.1002/hep.25569.

10. Shen J, Wang S, Zhang YJ, Wu HC, Kibriya MG, Jasmine F, et al. Exploring genome-wide DNA methylation profiles altered in hepatocellular carcinoma using Infinium HumanMethylation 450 BeadChips. Epigenetics. 2013;8(1):34-43. doi:10.4161/epi.23062.

11. Song MA, Tiirikainen M, Kwee S, Okimoto G, Yu H, Wong LL. Elucidating the landscape of aberrant DNA methylation in hepatocellular carcinoma. PLoS One. 2013;8(2):e55761. doi:10.1371/journal.pone.0055761.

12. Stefanska B, Huang J, Bhattacharyya B, Suderman M, Hallett M, Han ZG, et al. Definition of the landscape of promoter DNA hypomethylation in liver cancer. Cancer Res. 2011;71(17):5891-903. doi:10.1158/0008-5472.CAN-10-3823.

13. Udali S, Guarini P, Ruzzenente A, Ferrarini A, Guglielmi A, Lotto V, et al. DNA methylation and gene expression profiles show novel regulatory pathways in hepatocellular carcinoma. Clin Epigenetics. 2015;7(1):43. doi:10.1186/s13148-015-0077-1.

14. Wang J, Jiang H, Ji G, Gao F, Wu M, Sun J, et al. High resolution profiling of human exon methylation by liquid hybridization capture-based bisulfite sequencing. BMC Genomics. 2011;12:597. doi:10.1186/1471-2164-12-597.

15. Gao F, Wang J, Ji G, Liu S, Yao Y, Wang T et al. Clustering of cancer cell lines using a promoter-targeted liquid hybridization capture-based bisulfite sequencing approach. Technol Cancer Res Treat. 2014. doi:10.7785/ tcrt.2012.500416. 
16. Weber M, Hellmann I, Stadler MB, Ramos L, Paabo S, Rebhan M, et al. Distribution, silencing potential and evolutionary impact of promoter DNA methylation in the human genome. Nat Genet. 2007;39(4):457-66. doi:10.1038/ng1990.

17. Mortazavi A, Williams BA, McCue K, Schaeffer L, Wold B. Mapping and quantifying mammalian transcriptomes by RNA-Seq. Nat Methods. 2008:5(7):621-8. doi:10.1038/nmeth.1226.

18. Wang J, Xia Y, Li L, Gong D, Yao Y, Luo H, et al. Double restriction-enzyme digestion improves the coverage and accuracy of genome-wide CpG methylation profiling by reduced representation bisulfite sequencing. BMC Genomics. 2013;14:11. doi:10.1186/1471-2164-14-11.

19. Hagemann S, Heil O, Lyko F, Brueckner B. Azacytidine and decitabine induce gene-specific and non-random DNA demethylation in human cancer cell lines. PLoS One. 2011;6(3):e17388. doi:10.1371/journal.pone.0017388.

20. Tao SF, Zhang CS, Guo XL, Xu Y, Zhang SS, Song JR, et al. Anti-tumor effect of 5-aza-2'-deoxycytidine by inhibiting telomerase activity in hepatocellular carcinoma cells. World J Gastroenterol. 2012;18(19):2334-43. doi:10.3748/ wjg.v18.i19.2334.

21. Wang $X$, Kuang $Y Y$, Hu XT. Advances in epigenetic biomarker research in colorectal cancer. World J Gastroenterol. 2014;20(15):4276-87. doi:10.3748/wjg.v20.115.4276.

22. Yang $G, X u Y, C$ Chen $X, H u$ G. IFITM1 plays an essential role in the antiproliferative action of interferon-gamma. Oncogene. 2007;26(4):594-603. doi:10.1038/sj.onc.1209807.

23. Huang H, Colella S, Kurrer M, Yonekawa Y, Kleihues P, Ohgaki H. Gene expression profiling of low-grade diffuse astrocytomas by CDNA arrays. Cancer Res. 2000;60(24):6868-74.

24. Abba MC, Drake JA, Hawkins KA, Hu Y, Sun H, Notcovich C, et al. Transcriptomic changes in human breast cancer progression as determined by serial analysis of gene expression. Breast Cancer Res. 2004;6(5):R499-513. doi:10.1186/bcr899.

25. Lee J, Goh SH, Song N, Hwang JA, Nam S, Choi IJ, et al. Overexpression of IFITM1 has clinicopathologic effects on gastric cancer and is regulated by an epigenetic mechanism. Am J Pathol. 2012;181(1):43-52. doi:10.1016/ j.ajpath.2012.03.027.

26. Jiang Y, Liang H, Guo W, Kottickal LV, Nagarajan L. Differential expression of a novel C-terminally truncated splice form of SMAD5 in hematopoietic stem cells and leukemia. Blood. 2000;95(12):3945-50.

27. Hata A, Lo RS, Wotton D, Lagna G, Massague J. Mutations increasing autoinhibition inactivate tumour suppressors Smad2 and Smad4. Nature. 1997;388(6637):82-7. doi:10.1038/40424.

28. Cheng KH, Ponte JF, Thiagalingam S. Elucidation of epigenetic inactivation of SMAD8 in cancer using targeted expressed gene display. Cancer Res. 2004;64(5):1639-46.

29. Imamura T, Takase M, Nishihara A, Oeda E, Hanai J, Kawabata M, et al. Smad6 inhibits signalling by the TGF-beta superfamily. Nature. 1997;389(6651):622-6. doi:10.1038/39355.

30. Topper JN, Cai J, Qiu Y, Anderson KR, Xu YY, Deeds JD, et al. Vascular MADs: two novel MAD-related genes selectively inducible by flow in human vascular endothelium. Proc Natl Acad Sci U S A. 1997;94(17):9314-9.

31. Soliman HH, Nagy H, Kotb N, Alm El-Din MA. The role of chemokine CC ligand 20 in patients with liver cirrhosis and hepatocellular carcinoma. Int J Biol Markers. 2012;27(2):e125-31. doi:10.5301/JBM.2012.9097.

32. Campbell AS, Albo D, Kimsey TF, White SL, Wang TN. Macrophage inflammatory protein-3alpha promotes pancreatic cancer cell invasion. J Surg Res. 2005;123(1):96-101. doi:10.1016/j.jss.2004.07.013.

33. Brand S, Olszak T, Beigel F, Diebold J, Otte JM, Eichhorst ST, et al. Cell differentiation dependent expressed CCR6 mediates ERK-1/2, SAPK/JNK, and Akt signaling resulting in proliferation and migration of colorectal cancer cells. J Cell Biochem. 2006;97(4):709-23. doi:10.1002/jcb.20672.

34. Gao F, Liu X, Wu XP, Wang XL, Gong D, Lu H, et al. Differential DNA methylation in discrete developmental stages of the parasitic nematode Trichinella spiralis. Genome Biol. 2012;13(10):R100. doi:10.1186/gb-2012-13-10-r100.

\section{Submit your next manuscript to BioMed Central and take full advantage of:}

- Convenient online submission

- Thorough peer review

- No space constraints or color figure charges

- Immediate publication on acceptance

- Inclusion in PubMed, CAS, Scopus and Google Scholar

- Research which is freely available for redistribution

Submit your manuscript at www.biomedcentral.com/submit 